\title{
Reducing all-cause mortality among patients with psychiatric disorders: a population-based study
}

\author{
Steve Kisely MD PhD, Neil Preston PhD, Jianguo Xiao MD PhD, David Lawrence PhD, Sandra Louise BSc, \\ Elizabeth Crowe MB ChB MSc
}

Competing interests: Steve Kisely has received grant funding from the Australian Rotary Health Fund. No other competing interests were declared.

This article has been peer reviewed.

Correspondence to: Steve Kisely, s.kisely @uq.edu.au

CMAJ 2013. DOI:10.1503 /cmaj.121077

\begin{abstract}
Background: Among patients with psychiatric disorders, there are 10 times as many preventable deaths from physical disorders as there are from suicide. We investigated whether compulsory community treatment, such as community treatment orders, could reduce all-cause mortality among patients with psychiatric disorders.
\end{abstract}

Methods: We conducted a population-based survival analysis of an inception cohort using record linking. The study period extended from November 1997 to December 2008. The cohort included patients from all communitybased and inpatient psychiatric services in Western Australia (state population $1.8 \mathrm{mil}$ lion). We used a 2-stage design of matching and Cox regression to adjust for demographic characteristics, previous use of health services, diagnosis and length of psychiatric history. We collected data on successive cohorts for each year for which community treatment orders were used to measure changes in numbers of patients, their characteristics and outcomes. Our primary outcome was 2-year all-cause mortality. Our secondary outcomes were 1and 3-year all-cause mortality.

Results: The study population included 2958 patients with community treatment orders (cases) and 2958 matched controls (i.e. patients with psychiatric disorders who had not received a community treatment order). The average age for cases and controls was 36.7 years, and $63.7 \%$ (3771) of participants were men. Schizophrenia and other nonaffective psychoses were the most common diagnoses $(73.4 \%)$ among participants. A total of 492 patients $(8.3 \%)$ died during the study. Cox regression showed that, compared with controls, patients with community treatment orders had significantly lower all-cause mortality at 1, 2 and 3 years, with an adjusted hazard ratio of 0.62 (95\% confidence interval $0.45-0.86$ ) at 2 years. The greatest effect was on death from physical illnesses such as cancer, cardiovascular disease or diseases of the central nervous system. This association disappeared when we adjusted for increased outpatient and community contacts with psychiatric services.

Interpretation: Community treatment orders might reduce mortality among patients with psychiatric disorders. This may be partly explained by increased contact with health services in the community. However, the effects of uncontrolled confounders cannot be excluded.
$\mathrm{M}$ ortality among patients with psychiatric disorders is higher than in the general population. ${ }^{1}$ Chronic physical disorders such as cardiovascular disease and cancer are the main causes of death in this population, with risks 10 times that of suicide; however, such causes receive far less attention than suicides. ${ }^{1,2}$ Patients with schizophrenia die 15-20 years earlier than people in the general population, a difference that has increased over time. ${ }^{1,3}$ Reasons for this difference include socioeconomic disadvantage, adverse effects of medication and reduced access to health care. ${ }^{4,5}$

There are limited data on possible interventions aimed at preventing such deaths, most of which stress regular monitoring of physical status, peer support and collaboration with primary care. ${ }^{6,7}$ One study from Victoria, Australia, found that patients on conditional release from hospital had lower mortality than expected when use of community care, age, sex, inpatient experience and diagnosis were taken into account. ${ }^{8}$ However, $10 \%$ of these patients had dementia or other diseases of the nervous system, and patients with these diagnoses made up 29\% of the deaths in the study. Dementia is not a typical indication for compulsory community treatment. In addition, Victoria has one of the highest levels of use of community treatment orders, about 60 per 100000 population; thus, those results may not 
be generalizable to other locales. ${ }^{8}$ Although the authors controlled for time at risk, death could occur from 1 day to 11 years after the index date; ${ }^{9}$ most evaluations of community treatment orders are limited to 1 or 2 years after the order is issued..$^{910}$ Finally, the results were not adjusted for patients' marital status, education, country of birth, indigenous status or use of health services before the introduction of community treatment orders. Adjusting for these variables could reduce the bias inherent in drawing cases and controls from the same jurisdiction given the difficulties in controlling for all possible reasons for issuing these orders once they have been introduced.

We sought to assess the impact of community treatment orders on 1-, 2-, and 3-year survival in Western Australia. Community treatment orders provide a legal framework within which patients with a serious mental disorder are required to accept psychiatric treatment while living outside hospital. These orders are used across both Canada and Australia, are of similar duration in both countries, and are clinician-initiated rather than court-ordered (in contrast to the United States). ${ }^{10,11}$ Unlike in Canada, patients in Australia can be given community treatment orders without having been previously admitted to hospital. In practice, patients in both countries spend similar amounts of time in hospital before being given a community treatment order. ${ }^{12}$

We focused on deaths from physical illness, rather than suicides, as these are the most common causes of preventable death among people with severe mental illness. ${ }^{1,2}$ We thought that patients with community treatment orders would have lower mortality because of improved engagement with health services, thus allowing greater monitoring and management of physical health. Greater engagement would be shown via increased outpatient contacts following the receipt of a community treatment order, which would influence any association between compulsory community treatment and mortality.

\section{Methods}

We performed a population-based record-linkage analysis of all patients with community treatment orders in Western Australia for a period of 11 years. The study was approved by the human research ethics committees of the University of Queensland, Griffith University and the Department of Health of Western Australia.

\section{Intervention}

Community treatment orders were introduced in Western Australia in November 1997. They are issued by an authorized medical practitioner and cannot exceed 3 months in the first instance. Orders can be extended to 6 months, after which a new order must be made. Community treatment orders require a person with a mental illness or disorder to follow a treatment plan, including adherence to any medications and psychiatric outpatient visits. ${ }^{11}$ Community treatment orders set out the terms under which a person must accept medication, therapy, rehabilitation and other services while living in the community. If a person breaches a community treatment order, the person may be taken to a mental health facility and given appropriate treatment, including medication.

In the case of a breach, the psychiatrist first informs the patient in writing of the situation. If they still fail to co-operate, they are sent an Order to Attend, a form ordering the patient to attend a mental health facility at a particular time for treatment. If the patients still fails to attend, the police are authorised to apprehend and take the patient to a facility to receive treatment, which can be given whether the patient agrees or not.

\section{Data sources}

We collected information on all residents of Western Australia given community treatment orders in the 11 years after the orders were implemented using linked administrative health data. ${ }^{6,13}$ These included data from the Mental Health Information System of psychiatric inpatient, outpatient and community contacts; the Mental Health Review Board database of compulsory psychiatric treatment; the Hospital Morbidity Data System; and mortality data. The Hospital Morbidity Data System includes information on all inpatient treatment, regardless of specialty, as a marker of general medical and surgical history.

\section{Selection of participants}

We selected an inception cohort of all patients given an initial community treatment order. Previous work suggested that about $85 \%$ of orders are given at discharge from hospital. ${ }^{12}$ We selected the same number of participants for the case and control groups, matched by age, sex, psychiatric diagnosis and date of discharge from hospital (i.e., the index date). For a small number of patients issued community treatment orders in the community, rather than on discharge from hospital, the start of the order was the index date used for matching with controls. Our study period extended from the implementation of community treatment orders in November 1997 to December 2008.

We first identified patients for the case group from the Mental Health Review Board and controls from the Mental Health Information System, then linked their records to the other databases. An initial matching stage helped to reduce 
type I error in the multivariate analyses. We extracted anonymized information on both groups covering up to 1 year before and 11 years after the index date, where available. This information included psychiatric and nonpsychiatric admissions, bed-days and outpatient contacts 1 year before the community treatment order was issued. In addition, we measured patients' use of health services before the implementation of community treatment orders in 1997 and the overall duration of psychiatric symptoms. We collected data on successive cases and controls for each year until December 2008 to measure changes in the numbers and characteristics of patients given community treatment orders.

\section{Primary outcome}

Our primary outcome was all-cause mortality at 2 years' follow-up. Most evaluations of community treatment orders have been restricted to 1 or 2 years of follow-up, because it may be difficult to attribute any effect to the intervention beyond that period. ${ }^{11,12}$ However, 2 years allows for sufficient time to detect an effect on mortality, particularly for chronic physical conditions.

\section{Statistical analysis}

We used matching, multivariate and propensity score techniques to adjust for sociodemographic factors, clinical features and previous psychiatric or medical history, as measured by health service contacts. We adjusted for use of psychiatric services before community treatment orders were implemented in 1997, and before the community treatment order was issued, in terms of psychiatric admissions (overall and involuntary), bed-days and outpatient contacts. For patients issued community treatment orders during the first year of implementation, these adjustments overlapped, requiring sensitivity analyses. We selected these variables, because previous studies have shown their association with either mortality or compulsory treatment. . $^{14-18}$

We regarded cases and controls as independent, because there was no reason to believe that their outcomes were correlated in any way. ${ }^{19}$

We initially examined associations using unadjusted odds ratios (ORs). We then compared time to readmission using Kaplan-Meier survival curves, using a Cox regression analysis to adjust for confounders such as sociodemo-

Table 1: Characteristics of patients with (case group) or without (control group) community treatment orders

\begin{tabular}{|c|c|c|c|}
\hline \multirow[b]{2}{*}{ Characteristic } & \multicolumn{2}{|c|}{ Group, no. (\%) } & \multirow[b]{2}{*}{$\begin{array}{l}\text { Crude OR } \\
(95 \% \mathrm{Cl})\end{array}$} \\
\hline & $\begin{array}{c}\text { Case } \\
(n=2958)\end{array}$ & $\begin{array}{l}\text { Control } \\
(n=2958)\end{array}$ & \\
\hline Age $>34 \mathrm{yr}$ & $1524(51.5)$ & $1498(50.6)$ & $1.03(0.93-1.15)$ \\
\hline Marital status always single & $1910(64.6)$ & $1827(61.8)$ & $1.13(1.02-1.26)$ \\
\hline Male sex & $1885(63.7)$ & $1886(63.8)$ & $0.99 \quad(0.89-1.10)$ \\
\hline Engaged in work, study or home duties & $508(17.2)$ & $709(24.0)$ & $0.66 \quad(0.58-0.75)$ \\
\hline Country of birth outside of Australia & $797(26.9)$ & $686(23.2)$ & $1.22(1.01-1.37)$ \\
\hline Aboriginal or Torres Strait status & $283 \quad(9.6)$ & $284 \quad(9.6)$ & $1.00 \quad(0.84-1.18)$ \\
\hline Rural residence & $298(10.1)$ & $302(10.2)$ & $0.98 \quad(0.83-1.17)$ \\
\hline \multicolumn{4}{|l|}{ Diagnosis } \\
\hline Psychiatric outpatient contacts before $1997>6$ & $855(28.9)$ & $714(24.1)$ & $1.28(1.14-1.44)$ \\
\hline$>1$ psychiatric admission in $1 \mathrm{yr}$ before CTO issued & $1680(56.8)$ & $1276(43.1)$ & $1.73(1.56-1.92)$ \\
\hline $\begin{array}{l}\geq 1 \text { involuntary inpatient admission in } 1 \mathrm{yr} \text { before CTO } \\
\text { issued }\end{array}$ & $2776(93.8)$ & $1471(49.7)$ & $\begin{array}{l}15.40(13.05- \\
18.21)\end{array}$ \\
\hline Psychiatric bed-days before index date $>30$ & $2030(68.6)$ & $932(31.5)$ & $4.29 \quad(4.26-5.31)$ \\
\hline Psychiatric outpatient contacts before index date $\geq 12$ & $1680(56.8)$ & $1276(43.1)$ & $1.73(1.56-1.92)$ \\
\hline Nonpsychiatric admissions $>1$ & $1842(62.3)$ & $1957(66.2)$ & $0.85 \quad(0.76-0.94)$ \\
\hline Psychiatric symptom duration $>5 \mathrm{yr}$ & 1466 (49.6) & $1397(47.2)$ & $1.10 \quad(0.99-1.22)$ \\
\hline
\end{tabular}


graphic characteristics, clinical features and use of health services in the year before the community treatment order was issued. If continuous variables were skewed, they were dichotomized about the median or 75th percentile, as appropriate, to ease interpretation. In addition, we measured patients' use of health services after the index date. Inpatient bed-days in psychiatric and nonpsychiatric units were assessed separately. Finally, we adjusted for the year in which the community treatment order was issued in case clinicians were targeting orders more effectively as they gained greater experience and familiarity with the system.

We constructed 3 regression models. In the first, all variables were forced into the model on the basis of being associated with the independent or other dependent variables, theoretically, or on univariate analysis. In the second, we ran a forward stepwise model in which variables were entered according to their association with allcause mortality until no more reached statistical significance. Only variables significantly associated with the outcome on multivariate analysis were included in the final run of this model. In the third model, we adjusted survival using a propensity score derived from all possible confounders. In all models, we tested significance using the Wald statistic.

We assessed absolute risk reduction by calculating the number needed to treat (NNT).

\section{Sensitivity analyses}

We performed sensitivity analyses on the effect of log-transforming all skewed continuous data. In addition, we analyzed all-cause mortality at 12 and 36 months after the index date, the latter being the maximum interval for which data were available for the whole sample, including those patients entering the study in 2008. We also investigated any differences in all-cause mortality over the years of community treatment order availability.

We restricted admissions during the year before the community treatment order was issued to those that were involuntary while keeping all of the other variables. We then reperformed all of the models, replacing use of health services in the year before the community treatment order was issued with use of health services before the implementation of community treatment orders in 1997 . We did this because there was an overlap between the 2 variables for

Table 2: Predictors of death within 2 years of receiving a community treatment order or discharge from hospital for patients without a community treatment order

\begin{tabular}{|c|c|c|c|c|}
\hline Characteristic & $\begin{array}{c}\text { Patients alive at } 2 \text { years' } \\
\text { follow-up, no. (\%) } \\
n=5753\end{array}$ & $\begin{array}{l}\text { Patients who died within } \\
2 \text { years' follow-up, no. (\%) } \\
\qquad n=163\end{array}$ & $\begin{array}{c}\text { Crude HR } \\
(95 \% \mathrm{Cl}) \\
\text { cases v. controls }\end{array}$ & $\begin{array}{c}\text { Adjusted HR* } \\
(95 \% \mathrm{Cl}) \\
\text { cases v. controls }\end{array}$ \\
\hline Age $>34 \mathrm{yr}$ & $2923(50.8)$ & $99(60.7)$ & $1.49(1.08-2.04)$ & $1.50(1.04-2.17) \dagger$ \\
\hline Marital status always single & $3647(63.4)$ & $90(55.2)$ & $0.72(0.53-0.98)$ & $0.75(0.53-1.07)$ \\
\hline Male sex & $3665(63.7)$ & $106(65.0)$ & $1.05(0.77-1.46)$ & $1.28(0.90-1.81)$ \\
\hline Post-secondary education & $426 \quad(7.4)$ & $16(9.8)$ & $1.36(0.81-2.27)$ & $1.20(0.70-2.03)$ \\
\hline $\begin{array}{l}\text { Engaged in work, study or home } \\
\text { duties }\end{array}$ & $1192(20.7)$ & $25(15.3)$ & $0.72(0.47-1.10)$ & $0.70(0.45-1.09)$ \\
\hline $\begin{array}{l}\text { Country of birth outside of } \\
\text { Australia }\end{array}$ & $1445(25.1)$ & $38(23.3)$ & $0.91(0.63-1.30)$ & $0.82(0.56-1.20)$ \\
\hline Aboriginal or Torres Strait status & $551 \quad(9.6)$ & $16 \quad(9.8)$ & $1.02(0.61-1.71)$ & $1.22(0.71-2.10)$ \\
\hline Rural residence & $593(10.3)$ & $7 \quad(4.3)$ & $0.39(0.18-0.84)$ & $0.45(0.21-0.98) \dagger$ \\
\hline Affective disorder & $826(14.4)$ & $24(14.7)$ & $1.03(0.66-1.58)$ & $0.93(0.59-1.45)$ \\
\hline Community treatment order & $2887(50.2)$ & $67(41.1)$ & $0.69(0.51-0.95)$ & $0.62(0.45-0.86) \dagger$ \\
\hline $\begin{array}{l}>1 \text { psychiatric admission in } 1 \mathrm{yr} \\
\text { before CTO issued }\end{array}$ & $2728(47.4)$ & 78 (47.9) & $1.02(0.74-1.39)$ & $1.14(0.82-1.58)$ \\
\hline $\begin{array}{l}\text { Psychiatric bed-days before index } \\
\text { date }>30\end{array}$ & $2892(50.3)$ & $96(58.9)$ & $1.42(1.03-1.94)$ & $0.94(0.67-1.30)$ \\
\hline $\begin{array}{l}\text { Psychiatric outpatient contacts } \\
\text { before index date } \geq 12\end{array}$ & $2871(49.9)$ & $85(52.1)$ & $1.10(0.80-1.49)$ & $1.54(1.09-2.17) \dagger$ \\
\hline Nonpsychiatric admissions $>1$ & $3675(63.9)$ & $124(76.1)$ & $1.78(1.24-2.56)$ & $1.94(1.34-2.82) \dagger$ \\
\hline Psychiatric symptom duration $>5 \mathrm{yr}$ & $2780(48.3)$ & $83(50.9)$ & $1.10(0.81-1.50)$ & $0.83(0.59-1.16)$ \\
\hline
\end{tabular}


patients given community treatment orders in the first year of implementation, and to reduce type I error. We also investigated cause of death, as well as 1- and 3-year all-cause mortality. Finally, we only included patients given a community treatment order in the community without having been admitted to hospital.

\section{Results}

We identified 2958 patients with community treatment orders from November 1997 to December 2008, and 2958 controls matched by age, sex and psychiatric diagnosis. The number of patients with community treatment orders per year varied between 221 and 324, equating to about 12 per 100000 population. Of the patients with community treatment orders we identified, $2398(81.1 \%)$ were given the orders on discharge from hospital, whereas 560 were issued orders in the community. The mean age of patients with community treatment orders was 36.7 (standard deviation [SD] 13.6) years, and 1885 (63.7\%) of them were men. The most common diagnoses among participants were schizophrenia and other nonaffective psychoses (73.4\%), followed by affective disorders (14.4\%).

Matching of cases and controls was successful, in that there were no significant differences in age, sex or diagnosis (Table 1). Patients in the case group were more likely than controls to have always been single or born outside of Australia; they were less likely to be engaged in work, study or home duties, or to have been admitted to hospital for nonpsychiatric reasons (a measure of physical comorbidity). In addition,

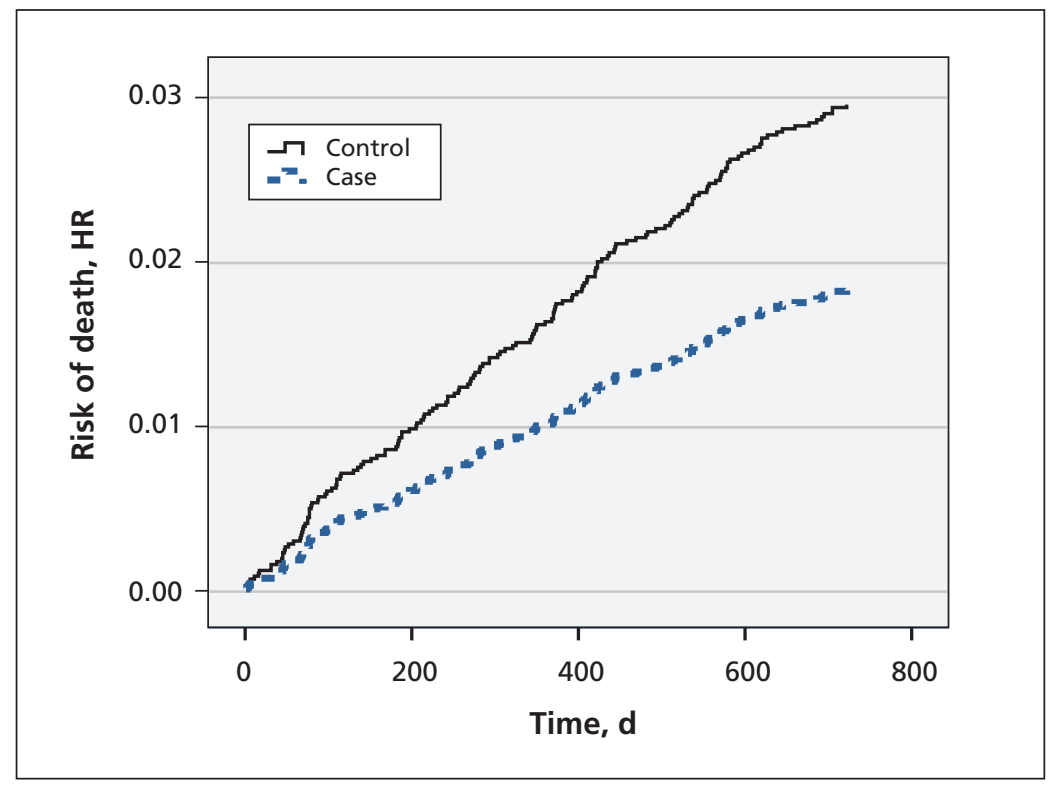

Figure 1: Mortality curves for patients with community treatment orders (cases) and their matched controls at 2-years' follow-up. HR = hazard ratio. patients with community treatment orders had greater overall use of health services, both during the year before their order was issued and before community treatment orders were implemented in 1997 (Table 1). There were no other differences between the two groups, including length of psychiatric history before being given a community treatment order.

During the 11 years of follow-up, 492 participants (cases and controls combined, 8.3\%) died. Only $6.5 \%$ of patients with community treatment orders died during this period (206/2958), compared with $9.6 \%$ of controls (286/2958) (OR $0.70,95 \%$ confidence interval [CI] $0.58-0.84$ ).

\section{Mortality at 2-years' follow-up}

A total of 163 patients died within 2 years of their index date. The causes of death included deliberate self-harm $(n=39)$, accidental injury $(n=35)$ and physical illness $(n=67)$. Of the deaths resulting from physical illness, 38 were due to cancer, cardiovascular disease or diseases of the central nervous system. The cause of death was unknown for 22 patients. Compared with controls, patients with community treatment orders were less likely to die in the 2 years after their index date (Kaplan-Meier survival analysis, log-rank $\chi^{2} 5.14, p=0.02$ ).

Compared with controls, patients with community treatment orders had nearly one-half of the all-cause risk of death (Wald 8.27, $p=0.004$ ) (Table 2 and Figure 1). Older patients had higher all-cause mortality. A greater number of psychiatric outpatient contacts and nonpsychiatric admissions were associated with increased risk of death, whereas rural residence-was associated with reduced risk (Table 2). We found similar results with stepwise regression, when we restricted the models to involuntary treatment before receiving a community treatment order while keeping all other variables, and when we used log-transformed data for all of the continuous variables. In addition, we found similar results using propensity score analysis (adjusted hazard ratio [HR] $0.63,95 \%$ CI $0.44-0.90$; Wald $6.55, p=0.01$ ).

Compared with patients in the control group, patients with community treatment orders had a significantly higher number of psychiatric admissions (0.31 [SD 4.40] v. 0.22 [SD 3.97]; $t$ test 8.55), bed-days (0.90 [SD 16.98] v. 0.41 [SD $10.71] ; t$ test 11.71$)$ and psychiatric outpatient contacts (10.23 [SD 8.79] v. 2.81 [SD 14.41]; $t$ test 20.23) (5914 degrees of freedom, all $p<$ $0.001)$. Including psychiatric admissions or beddays in the Cox regression had no effect on our results, but including psychiatric outpatient contacts did - the greater the number of outpatient 
contacts, the lower the subsequent all-cause mortality (adjusted HR 0.61, 95\% CI 0.50-0.75; Wald 27.94, $p<0.001)$. When we adjusted for psychiatric outpatient contacts, community treatment orders no longer showed an association with all-cause mortality (adjusted HR 0.79, 95\% CI 0.55-1.13; Wald 1.66, $p=0.19$ ).

In terms of subgroup analysis, removing deaths by suicide made no difference to our results (adjusted HR 0.55, 95\% CI 0.38-0.81; Wald $9.48, p=0.002$ ). By cause of death, the strongest effect was seen in deaths from cancer, cardiovascular disease or diseases of the central nervous system (adjusted HR 0.28, 95\% CI 0.130.61; Wald 9.21, $p=0.001$ ).

In terms of sensitivity analyses, replacing use of health services during the year before the community treatment order was issued with use of health services before community treatment orders were implemented in 1997 had no effect on the association between community treatment orders and reduced all-cause mortality (adjusted HR 0.67, 95\% CI 0.50-0.95; Wald 5.49, $p=$ 0.02). Restricting admissions during the year before the community treatment order was issued to only those that were involuntary also showed no effect on this association (adjusted HR 0.61, 95\% CI 0.43-0.87; Wald 7.39, $p=$ $0.01)$. We found similar results when we restricted our sample to patients who were given community treatment orders on discharge from hospital $(n=2398)$. In this population of patients and matched controls, there were 154 deaths (58 patients with community treatment orders, 96 controls; adjusted HR 0.65, 95\% CI 0.460.92; Wald 5.81, $p=0.01$ ).

We calculated the NNT to avoid a single death over a 2-year follow-up to be 102 .

\section{Mortality at 1 and 3 years' follow-up}

A total of 91 patients died during the year after they received their community treatment order (35 patients with community treatment orders, 56 controls). Patients with a community treatment order had a significant reduction in all-cause mortality (adjusted HR 0.58, 95\% CI 0.37-0.90; Wald $5.80, p=0.003$ ). The same was true for the 234 patients who died before the 3-year follow-up (103 patients with community treatment orders, 131 controls; adjusted HR 0.72, 95\% CI 0.550.95; Wald 5.50, $p=0.02$ ).

\section{Interpretation}

Community treatment orders were associated with a reduction in all-cause mortality after adjusting for confounders using matching, multivariate or propensity score techniques. These confounders included use of health services both before the community treatment order was issued and, where possible, the initial introduction of legislation implementing such orders. Our findings may have implications for Canada and other jurisdictions with similar types and levels of use of community treatment orders. ${ }^{11,20}$

Community treatment orders are controversial, with equivocal evidence of their effectiveness in reducing admissions to hospital or lengths of stay. ${ }^{9,10}$ Their only consistent effect is to increase the number of community and outpatient contacts, a process rather than outcome measure. ${ }^{9,10}$ It is possible that this is the mechanism by which mortality was reduced in our study. Increased contact with mental heath clinicians might have provided opportunities for identifying and managing comorbid physical illnesses, or for monitoring the presence of adverse effects of psychotropic medications known to increase the risk of chronic disease. This is consistent with our finding that the effect on mortality disappeared when we adjusted our model to include outpatient contacts after receiving a community treatment order. Increased engagement with psychiatric services must entail more than monitoring suicide risk, as community treatment orders had a greater effect on death from chronic physical illness than from other causes.

\section{Limitations}

We assessed psychiatric and physical morbidity only through records of patients' use of health services.

There may have been other confounders for which we were unable to adjust our models, which could explain why some patients were given compulsory community treatment. These potential confounders include lack of insight or treatment adherence, social disability, types of medication and characteristics of the treating teams or services. However, any bias should mean that patients with community treatment orders were more ill than patients in the control group, which is why they were given the orders in the first place. This rationale does not explain why all-cause mortality was lower among patients with community treatment orders than among controls, in spite of patients in the former group possibly having more severe illness.

Propensity score analysis as an alternative to multivariate techniques was limited by whether all possible confounders had been identified. ${ }^{21}$

The relative rarity of death in the overall sample meant that 102 people needed to be given a community treatment order to prevent 1 death in the first 2 years $(\mathrm{NNT}=102)$. However, 
this value is similar to those for reductions in 2year mortality after myocardial infarction for statins $(\mathrm{NNT}=94)$ and antiplatelet agents $(\mathrm{NNT}=153),{ }^{22}$ and better than many interventions to prevent suicide with NNTs of between 385 and $33000 .^{23}$

\section{Conclusion}

Community treatment orders might reduce mortality associated with preventable physical illness among patients with psychiatric disorders. Such a reduction may be partly explained by increased contact with health services in the community and better access to medically necessary treatments. Because community treatment orders in Western Australia resemble those in Canada, our results have implications for Canadian practice. Further research could establish whether patients given community treatment orders are more likely to receive appropriate care, because patients with psychiatric disorders are less likely to receive guideline-consistent management of physical illness. ${ }^{4,6,24,25}$

\section{References}

1. Tiihonen J, Lonnqvist J, Wahlbeck K, et al. 11-year follow-up of mortality in patients with schizophrenia: a population-based cohort study (FIN11 study). Lancet 2009;374:620-7.

2. Kisely S, Smith M, Lawrence D, et al. Mortality in individuals who have had psychiatric treatment: population-based study in Nova Scotia. Br J Psychiatry 2005;187:552-8.

3. Saha S, Chant D, McGrath J. A systematic review of mortality in schizophrenia: Is the differential mortality gap worsening over time? Arch Gen Psychiatry 2007;64:1123-31.

4. Kisely S, Smith M, Lawrence D, et al. Inequitable access for mentally ill patients to some medically necessary procedures. CMAJ 2007; 176:779-84.

5. Lawrence DM, Holman CD, Jablensky AV. Preventable physical illness in people with mental illness. Perth (AU): University of Western Australia; 2001

6. Lawrence D, Kisely S. Inequalities in healthcare provision for people with severe mental illness. J Psychopharmacol 2010;24: (Suppl 4):61-68.

7. Bates A, Kemp V, Isaac M. Peer support shows promise in helping persons living with mental illness address their physical health needs. Can J Commun Ment Health 2008;27:21-36.

8. Segal S P, Burgess PM. Effect of conditional release from hospitalization on mortality risk. Psychiatr Serv 2006;57:1607-13.

9. Preston NJ, Kisely S, Xiao J. Assessing the outcome of compulsory psychiatric treatment in the community: epidemiological study in Western Australia. BMJ 2002;324:1244.

10. Kisely SR, Campbell LA, Preston NJ. Compulsory community and involuntary outpatient treatment for people with severe mental disorders. Cochrane Database Syst Rev 2011;(2):CD004408.

11. Gray JE, McSherry BM, O'Reilly RL, et al. Australian and Canadian mental health acts compared. Aust N Z J Psychiatry 2010;44:1126-31.
12. Kisely S, Campbell LA, Preston NJ, et al. Can epidemiological studies assist in the evaluation of community treatment orders? The experience of Western Australia and Nova Scotia. Int J Law Psychiatry 2006;29:507-15.

13. Holman CD, Bass AJ, Rouse IL, et al. Population-based linkage of health records in Western Australia: development of a health services research linked database. Aust N Z J Public Health 1999;23:453-9.

14. Riecher A, Rossler W, Loffler W, et al. Factors influencing compulsory admission of psychiatric patients. Psychol Med 1991;21: 197-208.

15. Swartz MS, Swanson JW, Wagner HR, et al. Can involuntary outpatient commitment reduce hospital recidivism? Findings from a randomised trial with severely mentally ill individuals. Am J Psychiatry 1999; 156:1968-75.

16. Vaughan $\mathrm{K}$, McConaghy N, Wolf C, et al. Outpatient commitments: relationship to clinical care, medication compliance, behavioural disturbance and readmission. Aust N Z J Psychiatry 2000;34:801-8.

17. Steadman H J, Gounis K, Dennis D, et al. Assessing the New York City Involuntary Outpatient Commitment Pilot Program. Psychiatr Serv 2001;52:330-6.

18. Commander MJ, Cochrane R, Sashidharan SP, et al. Mental health care for Asian, black and white patients with non-affective psychoses: pathways to the psychiatric hospital, in-patient and after-care. Soc Psychiatry Psychiatr Epidemiol 1999;34: 484-91.

19. Schafer JL, Kang J. Average causal effects from nonrandomized studies: a practical guide and simulated example. Psychol Methods 2008;13:279-313.

20. Lawton-Smith S. A question of numbers: the potential impact of community-based treatment orders in England and Wales. London (UK) King's Fund; 2005. Available: www.kingsfund .org.uk/publications/question-numbers-community-based-treatment -orders-england-wales (accessed 2012 Jan. 13).

21. D'Agostino RB Jr. Propensity scores in cardiovascular research. Circulation 2007;115:2340-3.

22. Freemantle N, Cleland J, Young P, et al. $\beta$ Blockade after myocardial infarction: systematic review and meta regression analysis. BMJ 1999;318:1730-7.

23. Lewis G, Hawton K, Jones P. Strategies for preventing suicide. Br J Psychiatry 1997;171:351-4.

24. Druss BG, Bradford WD, Rosenheck RA, et al. Quality of medical care and excess mortality in older patients with mental disorders. Arch Gen Psychiatry 2001;58:565-72.

25. Kisely S, Campbell LA, Wang Y. Treatment of ischaemic heart disease and stroke in individuals with psychosis under universal healthcare. Br J Psychiatry 2009;195:545-50.

Affiliations: From the Department of Psychiatry, Community Health and Epidemiology (Kisely), Dalhousie University, Halifax, NS; The University of Queensland, School of Population Health (Kisely, Crowe), Herston; Griffith Health Institute (Kisely), Griffith University, Gold Coast, Queensland; and the Department of Health of Western Australia (Preston, Xiao, Louise), Perth; and the University of Western Australia (Lawrence), Perth, Western Australia

Contributors: Steve Kisely conceived of the idea for the study and the analysis plan. All of the authors assisted with the design of the study. Jianguo Xiao and Sandra Louise prepared the data files for analysis. All of the authors interpreted the analysis. Steve Kisely drafted the article. All of the authors revised the article for important intellectual content and approved the final version submitted for publication.

Funding: This work was supported by the Australian Rotary Health Fund (grant no. 007344). 